\title{
Glypican-3 is a useful diagnostic marker for a component of hepatocellular carcinoma in human liver cancer
}

\author{
HIROFUMI SHIRAKAWA ${ }^{1,3}$, TOSHIMITSU KURONUMA ${ }^{1}$, YOSHIKO NISHIMURA ${ }^{1}$, TAKAHIRO HASEBE ${ }^{2}$, \\ MASAYUKI NAKANO ${ }^{4}$, NAOTO GOTOHDA ${ }^{3}$, SHINICHIRO TAKAHASHI $^{3}$, TOSHIO NAKAGOHRI ${ }^{3}$, \\ MASARU KONISHI ${ }^{3}$, NOBUAKI KOBAYASHI ${ }^{5}$, TAIRA KINOSHITA ${ }^{3}$ and TETSUYA NAKATSURA ${ }^{1}$
}

\author{
${ }^{1}$ Section for Cancer Immunotherapy, Investigative Treatment Division, ${ }^{2}$ Pathology Division, Research Center for Innovative \\ Oncology, ${ }^{3}$ Hepato-Biliary Pancreatic Surgery Division, National Cancer Center Hospital East, 6-5-1 Kashiwanoha, Kashiwa, \\ 277-8577 Chiba; ${ }^{4}$ Department of Pathology, Tokyo Women's Medical University Yachiyo Medical Center, \\ 477-96 Owada-Shinden, Yachiyo, 276-8524 Chiba; ${ }^{5}$ Department of Organ Regulatory Surgery, \\ Ehime University Graduate School of Medicine, Shitsukawa, Toon, 791-0295 Ehime, Japan
}

Received September 10, 2008; Accepted October 27, 2008

DOI: 10.3892/ijo_00000190

\begin{abstract}
Primary liver cancers are classified into three types based on their morphology and cytogenetic characteristics hepatocellular carcinoma (HCC), intrahepatic cholangiocarcinoma (ICC) and combined hepatocellular and cholangiocarcinoma (CHC). It is often difficult to distinguish these liver tumors. Glypican-3 (GPC3) is serological and histochemical marker of hepatocellular carcinoma. In order to separate these three types of liver cancers, we analyzed the GPC3 expression in 85 liver resection specimens, including 46 HCCs, 28 ICCs and 11 CHCs. GPC3 immunohistochemical staining was used to distinguish HCC from ICC by comparing with the conventional biomarker, $\alpha$-fetoprotein (AFP). The immunostaining of GPC3 was identified in 78.3\% (36/46) of HCCs, $60 \%$ (9/15) of well differentiated, $88.9 \%$ $(16 / 18)$ of moderately differentiated and $84.6 \%(11 / 13)$ of poorly differentiated HCCs. It was negative in the ICCs. We confirmed that GPC 3 expression is specific to HCC component $(8 / 11,72.7 \%)$ but few samples also showed weakly in ICC component $(2 / 11,18.2 \%)$ of CHC sections among 11 cases compared with HCC biomarkers including
\end{abstract}

Correspondence to: Dr Tetsuya Nakatsura, Section for Cancer Immunotherapy, Investigative Treatment Division, Research Center for Innovative Oncology, National Cancer Center Hospital East, 6-5-1 Kashiwanoha, Kashiwa 277-8577, Japan

E-mail: tnakatsu@east.ncc.go.jp

Abbreviations: HCC, hepatocellular carcinoma; ICC, intrahepatic cholangiocarcinoma; $\mathrm{CHC}$, combined hepatocellular and chorangiocarcinoma; GPC3, glypican-3; AFP, $\alpha$-fetoprotein; HepPar1, hepatocyto paraffin 1; CK, cytokeratin; CC, cholangiocarcinoma; cp, component

Key words: hepatocellular carcinoma, intrahepatic cholangiocarcinoma, combined hepatocellular and chorangiocarcinoma, glypican-3, CK7, CK19, immunohistochemical analysis
AFP and hepatocyto paraffin 1 (HepPar1), and ICC biomarkers cytokeratin (CK) 7 and CK19. Three cases in which the macroscopic features resembled ICC did not express GPC3 even in the pathological HCC component. Most $(10 / 11,91 \%)$ of the pathological cholangiocarcinoma components in $\mathrm{CHC}$ showed positive staining for $\mathrm{CK} 7$ and CK19. The results of this study suggest that GPC3 is a biomarker that is sensitive and specific to HCC component of CHC, and CK7 and CK19 are markers for pathological cholangiocarcinoma component of $\mathrm{CHC}$.

\section{Introduction}

Liver cancer is one of the common malignancies that are rapidly increasing throughout the world. Primary liver cancers are classified into three types based on their morphology and cytogenetic characteristics, hepatocellular carcinoma (HCC), intrahepatic cholangiocarcinoma (ICC) and combined hepatocellular and cholangiocarcinoma (CHC). HCC is hepatocyto-origin, and ICC is from the epithelium of the intrahepatic bile duct. CHC is a rare type of liver cancer with features of both hepatocellular and biliary differentiation (1-3). The pathological structure of $\mathrm{CHC}$ is composed of hepatocellular element showing bile production, an intercellular bile canaliculi or trabecular growth pattern and cholangiocellular component showing mucin production or gland formation.

Because of their rapid growth rate and the lack of accurate ways of diagnosis in the early stages, the prognosis and the survival rate for liver cancer patients remain poor. Currently, ultrasound sonography (US), computed tomography (CT), magnetic resonance imaging (MRI), and histopathological examination for tumor biopsy are used for diagnosis. However, distinguishing the three different primary liver tumors is often a challenging task in diagnosis, for which immunohistochemical analysis for specific antigens is a helpful tool: $\alpha$-fetoprotein (AFP) and hepatocyto paraffin 1 (HepPar1) for HCC (4-8) and cytokeratin (CK) 7 and CK19 for ICC (9-11). 
Glypican-3 (GPC3) was discovered as a potential serological and histochemical marker whose expression is specific for HCC (12-16). GPC3 belongs to glypican family that is a group of heparan sulfate proteoglycans linked to the outer surface of cell membrane through a glycosylphosphatidylinositol anchor (17). In mammals, six members of GPCs have been reported, GPC1 to GPC6. GPCs are released from the cell surface by a lipase called Notum to regulate the signaling of Wnts, Hedgehogs, fibroblast growth factors (FGFs) and bone morphogenetic proteins (BMPs) (18-25). Depending on the cellular context, their function can be stimulatory or inhibitory activity, or signaling. The expression of GPC3 is detected in placenta and fetal liver, but not in other normal organs. During hepatic carcinogenesis, GPC3 have been reported to reappear in HCC and to be released into serum $(12,13,15,26)$. Its expression is also detected in melanoma (27-29). The functions of GPC3 in cancer cells are still unclear.

In this study, we examined whether immunohistochemical analysis for GPC3 can be used to distinguish HCC from ICC, if so, how effectively GPC3 can be detected, compared to other biomarkers that are conventionally used. We demonstrate that distinguishing HCC from ICC by detecting the expression of GPC3 enables more accurate diagnosis.

\section{Materials and methods}

Case selection. We selected 85 cases of liver tumors from the surgical pathology files from 1992 to 2006 of National Cancer Center Hospital East, Kashiwa, Chiba, Japan. The cases included 46 primary HCCs, 28 ICCs, and 11 CHCs that underwent hepatectomy. All identifiers were eliminated to protect patients' identities. Size of the tumor and any clinicopathologic factors (age, sex and grade of tumor) were matched between HCC and ICC. The 46 cases of HCCs occurred in 33 men and 13 women with a mean of age at 65.3 years (range, 44-80 years). HCC was subclassified into well $(n=15)$, moderately $(n=18)$, and poorly $(n=13)$ differentiated types according to the World Health Organization classification criteria. The 28 cases of ICC consisted of 18 men and 10 women. Their mean age was 65.7 years (range, 51-82 years). All 28 resected cases of ICC were confirmed by hematoxylin-eosin (H.E.) staining.

The 11 cases of $\mathrm{CHC}$ included 7 men and 4 women with a mean age of 62.5 years (range, 47-76 years). All CHCs were pathologically confirmed after surgery.

Tissue samples. Liver tissue sections were retrieved from the files of the Department of Pathology in our institution. All liver specimens were prepared from surgically resected tumors and adjacent parenchyma. They were fixed in $10 \%$ formalin and paraffinized for routine histological examination.

Immunohistochemical staining procedure. Six-micrometerthick sections were made from the paraffin-embedded blocks. Subsequently the sections were deparaffinized in xylene and rehydrated through ethanol to water. Endogenous peroxidase activity was blocked using $3 \% \mathrm{H}_{2} \mathrm{O}_{2}$ in methanol for $20 \mathrm{~min}$. For antigen retrieval, Sections were heated in $10 \mathrm{mM}$ citrate buffer ( $\mathrm{pH}$ 6.0) with microwave for $15 \mathrm{~min}$ in a water bath at $95^{\circ} \mathrm{C}$. Only for CK7 immunostaining, sections were digested by Proteinase K (DakoCytomation, Carpenteria, CA) for $5 \mathrm{~min}$ at room temprature. Slides were then allowed to cool down. The prediluted primary antibodies, monoclonal anti-GPC3 (dilution 1:300, 1G12; Biomosaics, Inc., Burlington, VT), anti-AFP (dilution 1:400, DakoCytomation), anti-HepPar1 (dilution 1:100, DakoCytomation), anti-CK7 (dilution 1:100, DakoCytomation), and CK19 (dilution 1:200, DakoCytomation) were added to cover each slide, and the slides were incubated for $2 \mathrm{~h}$ at room temperature. Slides were washed 3 times in phosphatebuffered saline (PBS)/Tween for $5 \mathrm{~min}$ each. Mouse Envision Polymer (DakoCytomation) was used as a secondary antibody for $30 \mathrm{~min}$ at room temperature followed by washes in PBS/Tween 3 times for 5 min each. Diaminobenzidine chromagen (DakoCytomation) was added to each slide and incubated for $2 \mathrm{~min}$. Slides were washed in distilled water, counterstained with hematoxylin and dehydrated in xylene. To analyze GPC3 expression, the immunohistochemical results were classified according to the number of positive cells as follows: -, negative $(<10 \%)$; \pm , weakly positive $(10-30 \%) ;+$ positive $(>30 \%)$. To validate the data in GPC3 as a marker for HCC, parallel staining for AFP of 46 cases were further analyzed. For $11 \mathrm{CHC}$ cases, AFP, HepPar1, CK 7 and CK19 were stained and compared with GPC3 staining pattern.

The slides were examined independently by 3 observers (Shirakawa H, Kuronuma T and Nakatsura T) and then collectively by 2 more pathologists (Hasebe T and Nakano M).

Statistical analysis. Differences in proportion were tested by the $\chi^{2}$ test. Differences in the means of each subgroup were tested using the Student's t-test. P-value of $<0.05$ was considered statistically significant.

\section{Results}

GPC3 was present in $80 \%$ of HCC and negative in ICC. In order to examine the levels and pattern of GPC 3 expression, 46 cases of HCC and 28 cases of ICC were immunohistochemically analyzed. GPC 3 was detected in 36 cases $(78 \%)$ of HCC (Fig. 1a), and no expression of GPC3 was found in any of the ICC patients (Fig. 1b). The GPC3 staining was diffused throughout (Fig. 1c) or localized in a granular pattern in the cytoplasm (Fig. 1d). In other cases, GPC3 was observed at the plasma membrane (Fig. 1e). Previously GPC3 is shown to bind to the cell membrane (16), however, those cases with membranous GPC 3 had staining in the cytoplasm as well, but there was no case of GPC3 located only at the plasma membrane. When sensitivity of GPC3 was evaluated, 36 cases $(78 \%)$ were positive for GPC3 when only 16 cases $(35 \%$; $\mathrm{P}<0.0001)$ were stained for AFP in HCC suggesting that GPC3 is more sensitive than AFP. Thus, GPC3 was confirmed to be specific and sensitive to HCC compared to AFP.

GPC3 expression increased in moderately and poorly differentiated HCC. In terms of GPC3 expression and tumor 

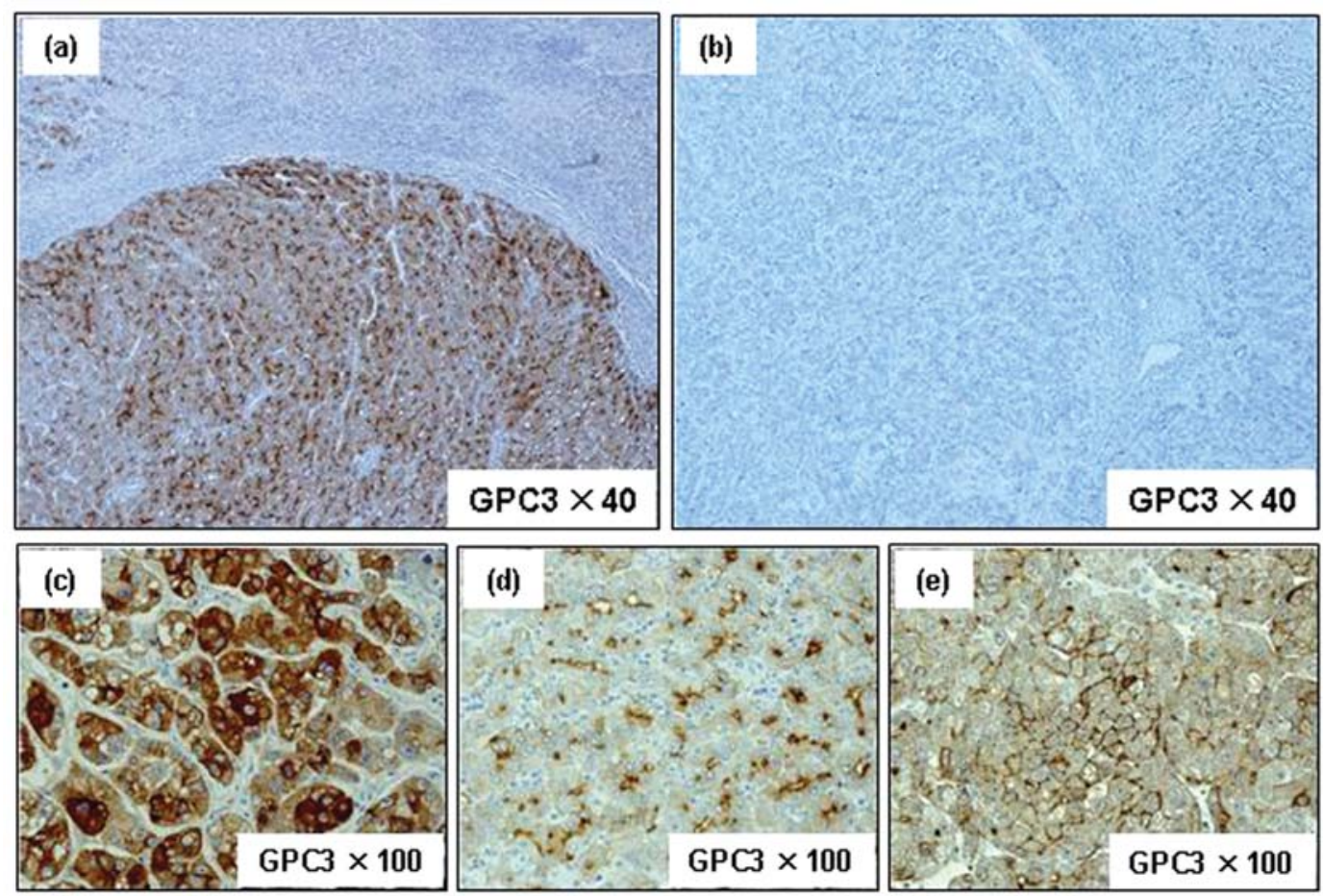

Figure 1. GPC3 expression was specific to HCC and absent in ICC. Immunohistochemical detection of GPC3 expression in HCC (a) and ICC (b) (magnification, x40). Immunostaining patterns of HCC: (c) diffuse in cytoplasm, gulanular in cytoplasm (d), and membranous (e).

Table I. Correlation of positive for GPC3 staining and tumor grade.

\begin{tabular}{|c|c|c|c|c|c|c|c|c|}
\hline \multirow[t]{3}{*}{ Grade of tumor } & \multicolumn{5}{|c|}{$\mathrm{HCC}$} & \multicolumn{2}{|c|}{ ICC } & \multirow{3}{*}{ P-value } \\
\hline & \multirow{2}{*}{$\begin{array}{l}\text { No. of } \\
\text { case }\end{array}$} & \multicolumn{4}{|c|}{ GPC3 } & \multirow{2}{*}{$\begin{array}{l}\text { No. of } \\
\text { case }\end{array}$} & \multirow{2}{*}{$\begin{array}{c}\text { GPC3 } \\
\text { positivity }\end{array}$} & \\
\hline & & - & \pm & + & positivity & & & \\
\hline Well-differentiated & 15 & 6 & 5 & 4 & $9(60 \%)$ & 8 & $0(0 \%)$ & \\
\hline Moderately differentiated & 18 & 2 & 4 & 12 & $16(89 \%)$ & 10 & $0(0 \%)$ & \\
\hline Poorly differentiated & 13 & 2 & 5 & 6 & $11(85 \%)$ & 10 & $0(0 \%)$ & \\
\hline Total & 46 & & & & $36(78 \%)$ & 28 & $0(0 \%)$ & $<0.0001$ \\
\hline
\end{tabular}

-, negative $(<10 \%)$; \pm , weakly positive $(10-30 \%) ;+$, positive $(>30 \%)$.

differentiation level, GPC3 was expressed in 9 (60\%) of 15 well differentiated, $16(89 \%)$ of 18 moderately differentiated and in $11(85 \%)$ of 13 poorly differentiated HCC (Table I). AFP was expressed in $3(20 \%)$ of 15 well differentiated, 6 $(33 \%)$ of 18 moderately differentiated and in $7(54 \%)$ of 13 poorly differentiated HCC (data not shown). The expression level of GPC3 was lower in well differentiated HCC than in the other HCC grades, though the difference was not statistically significant (well- vs. moderately differentiated: $\mathrm{P}=0.054$, well- vs. poorly differentiated: $\mathrm{P}=0.150$ ). Thus, GPC3 expression is also a good indicator for malignancy levels.

GPC3 expression was observed specifically in pathological HCC component in $\mathrm{CHC}$. There are discrepancies between preoperative diagnosis and pathological findings for $\mathrm{CHC}$ patients. Diagnostic results and the expression of tumor markers of 11 CHC patients are summarized in Table II. Initial diagnosis was carried out by H.E. staining. Among these 11 patients, 7 patients (63.6\%) were diagnosed as HCC and $3(27.3 \%)$ were ICC. Only 1 patient (9\%) of the $11 \mathrm{CHC}$ was correctly diagnosed as $\mathrm{CHC}$. To seek the possibility to use GPC3 immunostaining to detect HCC component (cp) in CHC, combination of antibodies against GPC3, AFP, HepPar1, CK7 and CK17 were used. In addition to AFP, HepPar1 is frequently used as marker for HCC (4-8) and CK 7 and CK19 for ICC (9-11).

Among $11 \mathrm{CHC}$ cases, 4 cases preoperatively diagnosed as HCC were chosen to represent the collision and transitional type of CHCs based on the macroscopic features 
Table II. Correlation of immunostaining varieties and pathological components of CHC.

\begin{tabular}{|c|c|c|c|c|c|c|c|c|c|c|c|c|}
\hline \multirow[t]{2}{*}{$\begin{array}{l}\text { Pt. } \\
\text { no. }\end{array}$} & \multirow[t]{2}{*}{$\begin{array}{l}\text { Preoperative } \\
\text { diagnosis }\end{array}$} & \multirow[t]{2}{*}{$\begin{array}{c}\text { Macroscopic } \\
\text { diagnosis }\end{array}$} & \multicolumn{5}{|c|}{$\begin{array}{l}\text { Pathological hepatocellular } \\
\text { carcinoma component }\end{array}$} & \multicolumn{5}{|c|}{$\begin{array}{l}\text { Pathological cholangiocarcinoma } \\
\text { component }\end{array}$} \\
\hline & & & GPC3 & AFP & HepPar1 & CK7 & CK19 & GPC3 & AFP & HepPar1 & CK7 & CK19 \\
\hline 1 & $\mathrm{HCC}$ & $\mathrm{CHC}$ & + & + & - & + & + & - & - & - & - & - \\
\hline 2 & HCC & $\mathrm{HCC}$ & + & - & - & - & - & - & - & + & + & + \\
\hline 3 & $\mathrm{HCC}$ & $\mathrm{HCC}$ & + & - & + & - & - & \pm & - & - & + & + \\
\hline 4 & $\mathrm{CHC}$ & $\mathrm{HCC}$ & + & + & + & - & - & \pm & - & - & + & + \\
\hline 5 & $\mathrm{HCC}$ & $\mathrm{CHC}$ & + & - & + & - & - & - & - & - & + & + \\
\hline 6 & HCC & $\mathrm{CHC}$ & + & - & - & - & - & - & - & + & + & + \\
\hline 7 & ICC & $\mathrm{CHC}$ & \pm & - & - & \pm & + & - & - & - & + & + \\
\hline \multirow[t]{3}{*}{8} & $\mathrm{HCC}$ & $\mathrm{HCC}$ & + & + & - & - & - & - & + & - & + & + \\
\hline & \multicolumn{2}{|c|}{ Total \pm} & $8 / 8$ & $3 / 8$ & $3 / 8$ & $3 / 8$ & $2 / 8$ & $2 / 8$ & $1 / 8$ & $2 / 8$ & $7 / 8$ & $7 / 8$ \\
\hline & \multicolumn{2}{|c|}{ positive rate $(\%)$} & 100 & 38 & 38 & 38 & 25 & 25 & 13 & 25 & 88 & 88 \\
\hline 9 & ICC & ICC & - & - & - & - & - & - & - & - & + & + \\
\hline 10 & HCC & ICC & - & - & - & + & \pm & - & - & - & + & + \\
\hline 11 & ICC & ICC & - & - & - & + & + & - & - & - & + & + \\
\hline \multicolumn{3}{|c|}{ Total \pm} & $0 / 3$ & $0 / 3$ & $0 / 3$ & $2 / 3$ & $2 / 3$ & $0 / 3$ & $0 / 3$ & $0 / 3$ & $3 / 3$ & $3 / 3$ \\
\hline \multicolumn{3}{|c|}{ positive rate $(\%)$} & 0 & 0 & 0 & 67 & 67 & 0 & 0 & 0 & 100 & 100 \\
\hline
\end{tabular}

-, negative $(<10 \%)$; \pm , weakly positive $(10-30 \%) ;+$, positive $(>30 \%)$; HCC, hepatocellular carcinoma; ICC, intrahepatic cholangiocarcinoma; CHC, combined hepatocellular and cholangiocarcinoma; GPC3, glypican-3; AFP, $\alpha$-fetoprotein; HepPar1, hepatocyto-paraffin 1; $\mathrm{CK}$, cytokeratin; CC, cholangiocarcinoma.

in cut surface. In Fig. 2, macroscopic observation and the immunostained histological sections are shown. These sections include 2 elements with pathological HCC cp forming bile production and trabecular growth pattern by eosinophilic staining and cholangiocarcinoma (CC) cp forming mucin production or gland formation by basophilic staining. Cases 1-8 were GPC3 positive, and cases 9-11 were negative for GPC3 in the HCC cp. Macroscopic, histological and immunohistochemical features of cases 2, 6, 8 and 10 are shown in Fig. 2a, b, c and d. Case 2 had greenish white and yellow nodules within the same tumor mass in the cut surface. HCC subtypes such as simple nodular and confluent multinodular type exist. Case 2 exhibited the features of HCC with multinodular type (Fig. 2a-i). Pathological diagnosis by H.E. staining revealed CHC pathologically (Fig. 2a-ii and -iii), which was so-called 'collision'-type tumor as reported by Goodman et al (30). A 'collision'-type tumor is coincidental occurrence of HCC and CC within the same tumor mass (31). GPC3 was positive (Fig. 2a-iv), but AFP and HepPar1 were not detected in HCC cp (Fig. 2a-v and -vi). Although HepPar 1 is generally used as HCC marker, it was unexpectedly stained in $\mathrm{CC}$ region as well as CK7 and CK19 (Fig. 2a-vii and-viii).

Case 6 showed pale and lobulated phenotype in the cut surface macroscopically (Fig. 2b-i), and pathological diagnosis was also confirmed by H.E. staining (Fig. 2b-ii and -iii). This was so-called 'transitional' type tumor (30). A 'transitional' type tumor has an area of HCC that appears to transform into CC (31). GPC3 was stained in pathological
HCC cp (Fig. 2b-iv) where AFP was negative (Fig. 2b-v). The HCC region was surrounded by pathological CC $\mathrm{cp}$ with the staining for CK7 (Fig. 2b-vii). HepPar1 and CK19 were detected in the same region with $\mathrm{CC} \mathrm{cp}$ (Fig. 2b-vi and -viii). HepPar1 stained the CC cp as in case 2. The immunoreactivity of CK19 was not consistent with that of CK7.

Case 8 was diagnosed as HCC similarly to cases 2 and 6 , but mixed tumor masses with white and gray in the cut surface were observed (Fig. 2c-i and c-ii). Both GPC3 and AFP were positive in HCC cp (Fig. 2c-iv and -v). HepPar1 was stained in CC cp (Fig. 2c-vi). CK7 and CK19 were positive in CC cp (Fig. 2c-vii and-viii), especially CK19 was more specific for $\mathrm{CC}$ cp than CK7. These three cases (cases 2, 6 and 8) indicated that detecting GPC3 can compensate for AFP and enhance the ability to identify the presence of $\mathrm{HCC} \mathrm{cp}$ in $\mathrm{CHC}$.

Cases 9, 10 and 11 were negative for GPC3 expression in several tumors. Macroscopically, they had the features of ICC with irregular shaped, white solid tumor masses. As an example, case 10 is shown in Fig. 2d. Althogh case 10 was diagnosed as HCC preoperativerly, it showed macroscopic freatures of ICC with the presence of abundant fibrous stroma and indistinct tumor margin (Fig. 2d-i). This case was later diagnosed as $\mathrm{CHC}$ based on the pathological examination (Fig. 2d-ii and d-iii). GPC3, AFP and HepPar1 were not detected in either HCC cp or CC cp (Fig. 2d-iv, -v, and -vi). CK7 was stained diffusely in the tumor (Fig. 2dvii), and CK19 expression was more specific in CC cp than CK7 (Fig. 2d-viii). These 3 cases showed positive staining 

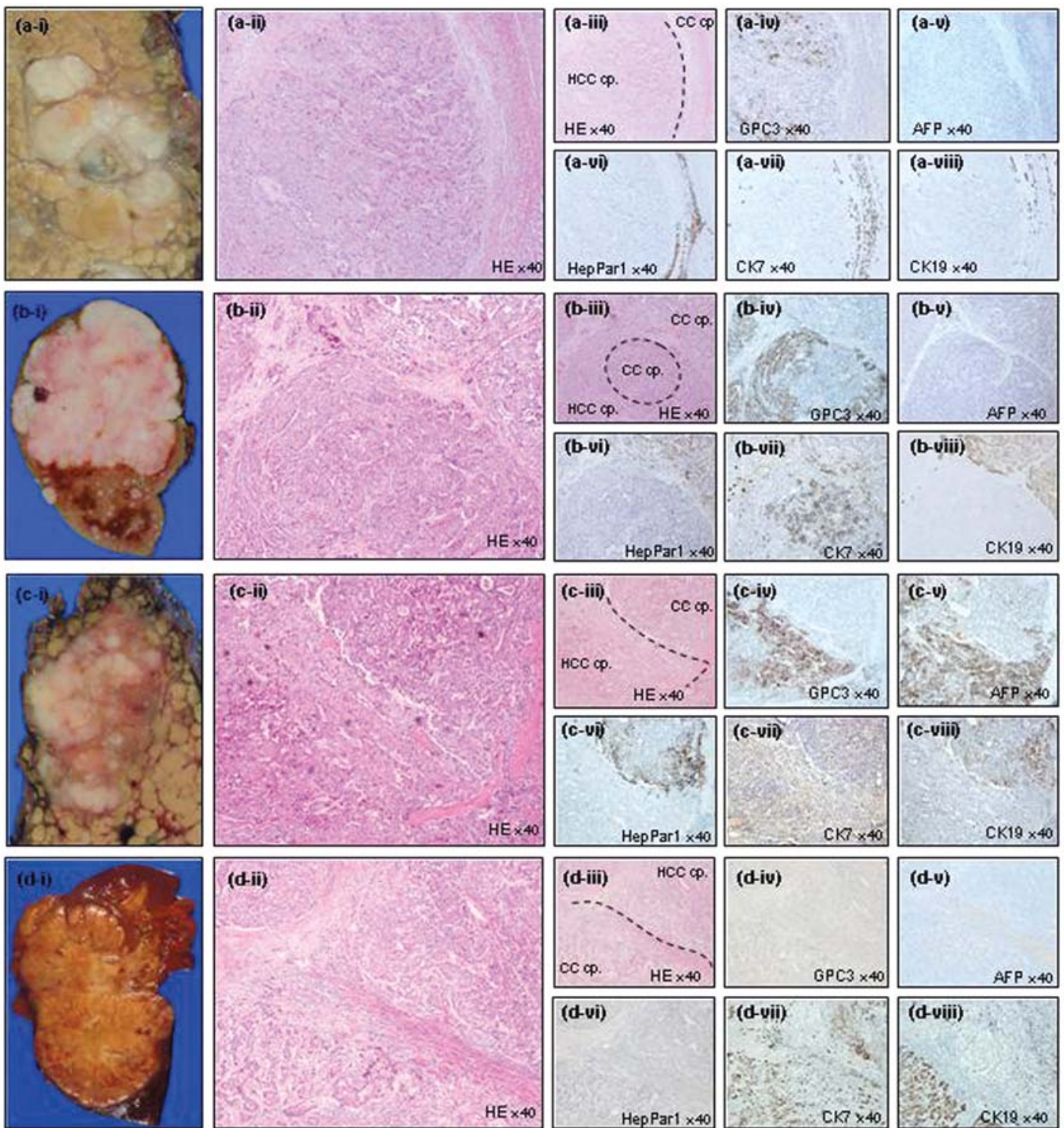

$\mathrm{HCC} \mathrm{cp} ., \mathrm{HCC}$ component; $\mathrm{CC} \mathrm{cp}$., cholangiocarcinoma component;

Figure 2. Macroscopic, histological and immunohistochemical features of four cases of CHC, a, case 2; b, case 6; c, case 8; d, case 10 in Table II. (a-i) Macroscopic feature in cut surface of case 2 tumor. (a-ii) The histological structure can be also devided into 2 types. HCC component showed expansive growth oppressing the cholangiocarcinoma component. (a-iii) Collision border between hepatocellular carcinoma and cholangiocarcinoma component are indicated as dots. The tumor cells within mainly hepatocellular carcinoma component showed only expression of GPC3 (a-iv) without expression of AFP (a-v). In the opposite side, the glandular area with cholangiocarcinoma component shows HepPar1 (a-vi), CK7 (a-vii) and CK19 expression (a-viii). (b-i) Case 6 shows macroscopic $\mathrm{CHC}$ feature in tumor cut surface that was suspected out HCC preoperatively. (b-ii) The histological cholangiocarcinoma component forming trabeculae with columnar appearance was surrounded by HCC component forming hepatoid structure. (b-iii) A dotted line is a boundary of HCC in the H.E. staining. The tumor cells within transitional region were positive for GPC3 (b-iv), CK 7 (b-vii) and CK 19 (b-viii). The difference was recognized between hepatocellular carcinoma component and cholangiocarcinoma component because GPC3 positive area encircled the CK7 area. The expressions of AFP (b-v) and HepPar1 (b-vi) were not observed. (c-i) Though case 8 was also suspected to be HCC preoperatively, the macroscopic features showed atypical HCC with mixed white and gray and indistinct tumor border. (c-ii) The cholangiocarcinoma component was obviously composed of structural gland formation. (c-iii) Collision area was distinguished histopathologically by a dotted line. The tumor cells of HCC component showed not only GPC3 (c-iv) but also AFP expression (c-v). In the glandular area of cholangiocarcinoma component, HepPar1 was expressed (c-vi), but CK7 not at all (c-vii) and CK19 shows weak positive expression (c-viii). (d-i) Case 10 shows macroscopic ICC features in tumor cut surface that was suspected as HCC preoperatively. (d-ii) The histological structure can be devided into 2 types with cholangiocarcinoma component forming trabeculae with columnar appearance and HCC component forming hepatocellular structures. (d-iii) A dotted line is a boundary of HCC in the H.E. GPC3 (d-iv), AFP (d-v) and HepPar1 (d-vi) were not stained, but CK7 (d-vii) and CK19 (d-viii) stained the cholagiocarcinoma component.

for CK7 and CK19 in CC cp, but not AFP or HepPar1 in HCC cp. Therefore, accuracy of CHC diagnosis can be achieved by combination of multiple tumor markers in addition to morphological characteristics: GPC3 that is specific for pathological HCC cp of CHC, and CK7 and CK19 that are specific for pathological CC cp of CHC.

\section{Discussion}

The diagnosis for HCC, ICC and $\mathrm{CHC}$ has been routinely performed by histopathological examination. Additionally, diagnosis of HCC is done by supplementary immunohistochemical analysis for AFP and HepPar1. Until now, though 
the sensitivity is limited, AFP has been regarded as the most useful marker for HCC (4,32-34). HepPar1 is also widely used for HCC to distinguish between primary HCC and ICC. However, both markers are limited for the ability to discriminate different levels of malignancy in HCC because its sensitivity drops substantially in poorly differentiated HCC, and it does not discriminate between benign and malignant liver cancers (35). As these biomarkers frequently results in misdiagnosis, in this study, we showed that GPC3 is more sensitive to detect HCC compared to AFP. Due to the fact that GPC3 was downregulated in ICC (36), GPC3 may help to separate HCC from ICC.

$\mathrm{CHC}$ is the least common primary cancer of the liver but followed by an aggressive growth, it tends to metastasize to many organs leading to significantly poorer prognosis than HCC and ICC $(31,37,38)$. Correct diagnosis leads to both appropriate treatment and better outcome for the patients. Nishie, et al reported that one third (nine of 27 cases) of patients with $\mathrm{CHC}$ were correctly diagnosed by enhanced computed tomography (39). In our study, only one of the 11 (9.1\%) patients with $\mathrm{CHC}$ was correctly diagnosed before operation without fine needle aspiration biopsy. The difficulty to pathologically distinguish CHC from HCC and ICC comes from glandular or pseudoglandular structures in HCC and solid or trabecular patterns in CC $(37,38)$. We believe that combination with histopathological examination with GPC3 immunostaining and radiological examination can bring an accurate diagnosis and improved clinical therapies for the patients leading to a better prognosis.

We showed that the immunostaining for GPC3 is specific for HCC patients and not detected in ICC patients. This confirmed that detecting GPC3 may improve the method to diagnose $\mathrm{CHC}$. Of the 11 cases of $\mathrm{CHC}, 8$ displayed GPC3 expression in restricted area of $\mathrm{HCC} \mathrm{cp}$. We demonstarated that immunohistochemical staining of GPC3 in liver tumor helps to recognize the pathological HCC cp more precisely. GPC3 expression was observed with high frequency in the HCC cp compared with AFP and HepPar1. HepPar1 was unexpectedly stained in CC cp, but this has been observed previously as well $(7,40)$. This could be due to a transition from HCC to ICC where HepPar1 is one of the molecules that is downregulated at later stages in the process. CK7 and CK19 have been already reported as good markers of biliary epithelial differentiation (41). These were highly expressed in pathological CC cp $(10 / 11,91 \%)$ in CHC. The positive immunoreactivity of CK19 was more distinct than that of CK7 whose staining was weaker. Our immunohistochemical data disclosed that GPC3 can be a better marker specific for HCC leading to a better confirmation for HCC component of $\mathrm{CHC}$ as well as for HCC. Moreover, it provided evidence of the biologic behavior of such combined tumors, which are phenotypically and genetically leaning toward either ICC with predominant biliary differentiation or HCC with hepatocellular differentiation $(42,43)$.

Employing multiple tumor markers may also allow the accurate diagnosis of $\mathrm{CHC}$ containing both hepatocellular and biliary differentiation. Concerning sensitivity and specificity, the combination of GPC3 for HCC cp and CK19 for ICC cp seems to be useful in the diagnosis of liver cancer.
For $\mathrm{CHC}$, GPC3 positive/CK19 negative profile suggests HCC, GPC3 positive/CK19 positive indicates CHC, and GPC3 negative/CK19 positive essentially rules out HCC and suggests the possibility of $\mathrm{CC}$ or $\mathrm{CHC}$.

We developed a new anti-cancer immunotherapy with GPC3 as a target (44-47), and the phase I clinical trial of GPC3-derived peptide vaccination for advanced HCC is now on going. Because this new immunotherapy is not indicated for ICC, immunohistochemical staining of GPC3 is a useful method to select eligible patients. Furthermore, if $\mathrm{CHC}$ would be justified as a target of our immunotherapy in future, immunohistochemical analysis for GPC3 expression is indispensable for the process of patient selection.

GPC3 is expressed in the group of cells that are AFPpositive and/or CK7/19-positive in injured livers with activation of oval cell compartment; an indication for liver repair and regeneration (48). In addition, CK7, CK19 and AFP are frequently expressed in biliary epithelial cells $(49,50)$ and in immature fetal hepatoblasts $(51,52)$. Liver progenitor cells originate from the canal of Hering, lined by both hepatocytes and biliary ductular epithelial cells (53). It is not clear whether GPC3 is expressed in hepatic embryonic progenitor cells or cancer stem cells, but GPC3 may be a marker for hepatic progenitor/stem cells. In CHC cases of 2, 3 and 4, GPC3, CK7 and CK19 coincided in the regions of HCC and CC. Although HCC and ICC are two different kinds of primary liver malignancies arising from different cell types as hepatocytes and cholangiocytes, co-localization of GPC3 and CK7/19 suggest that the $\mathrm{CHC}$ is originated from progenitor or oval cell. In addition, case 6 showed an HCC lesion with GPC3 positive immunostaining surrounded by CC (Fig. 2b). This finding suggests that GPC3-positive HCC tumor cells are derived from GPC3-negative CC mass. Moreover, we predict from the fact that GPC 3 is expressed in embryonic liver and downregulated after birth in normal liver but reappears in cancer is due to its regulatory role in proliferative and dedifferentiated cells, like cancer cells that acquired a progenitor- or cancer stem cell-like characteristics.

In summary, we confirmed that GPC3 is a marker sensitive and specific for HCC, but not ICC. Moreover, we revealed that GPC3 was expressed specifically in the HCC $\mathrm{cp}$ in the CHC. Therefore, GPC3 is a molecule that is significant not only in clinical but also biological field. It is clinically an important biomarker that can be used for accurate diagnosis leading to a better treatment and prognosis. Also, biologically, it may be an indicator for the identity and the origin of the cancer cells.

\section{Acknowledgments}

This study was supported in part by Health and Labor Sciences Research Grants for Research on Hepatitis from the Ministry of Health, Labor, and Welfare, Japan, and a grant-in-aid for the Third-Term Comprehensive 10-Year Strategy for Cancer Control from the Ministry of Health, Labour and Welfare, Japan. Foundation for Promotion of Cancer Research in Japan, Japan Research Foundation for Clinical Pharmacology and Research Resident Fellowship from the Foundation for Promotion of Cancer Research, Japan (H.S.). We thank Dr Chinatsu Kojima (Section for Cancer Immunotherapy, Investigative Treatment Division, 
Research Center for Innovative Oncology, National Cancer Center Hospital East) for technical assistance.

\section{References}

1. Aoki K, Takayasu K, Kawano T, et al: Combined hepatocellular carcinoma and cholangiocarcinoma: clinical features and computed tomographic findings. Hepatology 18: 1090-1095, 1993

2. Ng IO, Shek TW and Nicholls J and Ma LT: Combined hepatocellular-cholangiocarcinoma: a clinicopathological study. J Gastroenterol Hepatol 13: 34-40, 1998.

3. Liu CL, Fan ST, Lo CM, et al: Hepatic resection for combined hepatocellular and cholangiocarcinoma. Arch Surg 138: 86-90, 2003.

4. Brumm C, Schulze C, Charels K, Morohoshi T and Kloppel G: The significance of alpha-fetoprotein and other tumour markers in differential immunocytochemistry of primary liver tumours. Histopathology 14: 503-513, 1989.

5. Wennerberg AE, Nalesnik MA and Coleman WB: Hepatocyte paraffin 1: a monoclonal antibody that reacts with hepatocytes and can be used for differential diagnosis of hepatic tumors Am J Pathol 143: 1050-1054, 1993.

6. Minervini MI, Demetris AJ, Lee RG, Carr BI, Madariaga J and Nalesnik MA: Utilization of hepatocyte-specific antibody in the immunocytochemical evaluation of liver tumors. Mod Pathol 10: 686-692, 1997

7. Leong AS, Sormunen RT, Tsui WM and Liew CT: Hep Par 1 and selected antibodies in the immunohistological distinction of hepatocellular carcinoma from cholangiocarcinoma, combined tumours and metastatic carcinoma. Histopathology 33: 318-324, 1998

8. Lau SK, Prakash S, Geller SA and Alsabeh R: Comparative immunohistochemical profile of hepatocellular carcinoma, cholangiocarcinoma, and metastatic adenocarcinoma. Human Pathol 33: 1175-1181, 2002.

9. Maeda T, Kajiyama K, Adachi E, Takenaka K, Sugimachi K and Tsuneyoshi M: The expression of cytokeratins 7, 19, and 20 in primary and metastatic carcinomas of the liver. Mod Pathol 9: 901-909, 1996

10. Sasaki A, Kawano K, Aramaki M, Nakashima K, Yoshida T and Kitano S: Immunohistochemical expression of cytokeratins in intrahepatic cholangiocarcinoma and metastatic adenocarcinoma of the liver. J Surg Oncol 70: 103-108, 1999.

11. Shimonishi T, Miyazaki K and Nakanuma Y: Cytokeratin profile relates to histological subtypes and intrahepatic location of intrahepatic cholangiocarcinoma and primary sites of metastatic adenocarcinoma of liver. Histopathology 37: 55-63, 2000.

12. Capurro M, Wanless IR, Sherman M, et al: Glypican-3: a novel serum and histochemical marker for hepatocellular carcinoma. Gastroenterology 125: 89-97, 2003

13. Nakatsura T, Yoshitake Y, Senju S, et al: Glypican-3, overexpressed specifically in human hepatocellular carcinoma, is a novel tumor marker. Biochem Biophys Res Commun 306 : $16-25,2003$

14. Sung YK, Hwang SY, Park MK, et al: Glypican-3 is overexpressed in human hepatocellular carcinoma. Cancer Sci 94: 259-262, 2003

15. Hippo Y, Watanabe K, Watanabe A, et al: Identification of soluble NH2-terminal fragment of glypican-3 as a serological marker for early-stage hepatocellular carcinoma. Cancer Res 64: 2418-2423, 2004

16. Yamauchi N, Watanabe A, Hishinuma M, et al: The glypican 3 oncofetal protein is a promising diagnostic marker for hepatocellular carcinoma. Mod Pathol 18: 1591-1598, 2005.

17. Filmus J: The contribution of in vivo manipulation of gene expression to the understanding of the function of glypicans. Glycoconj J 19: 319-323, 2002.

18. De Cat B, Muyldermans SY, Coomans C, et al: Processing by proprotein convertases is required for glypican-3 modulation of cell survival, Wnt signaling and gastrulation movements. J Cell Biol 163: 625-635, 2003.

19. Capurro MI, Shi W, Sandal S and Filmus J: Processing by convertases is not required for glypican-3-induced stimulation of hepatocellular carcinoma growth. J Biol Chem 280: 41201-41206, 2005.

20. Capurro MI, Xiang YY, Lobe C and Filmus J: Glypican-3 promotes the growth of hepatocellular carcinoma by stimulating canonical Wnt signaling. Cancer Res 65: 6245-6254, 2005.
21. Song HH, Shi W, Xiang YY and Filmus J: The loss of glypican3 induces alterations in Wnt signaling. J Biol Chem 280: 2116-2125, 2005

22. Varma RR, Hector SM, Clark K, Greco WR, Hawthorn L and Pendyala L: Gene expression profiling of a clonal isolate of oxaliplatin-resistant ovarian carcinoma cell line A2780/C10. Oncol Rep 14: 925-932, 2005.

23. Filmus J, Capurro M and Rast J: Glypicans. Genome Biol 9: 224, 2008

24. Stigliano I, Puricelli L, Filmus J, Sogayar MC, Bal de Kier Joffe E and Peters MG: Glypican-3 regulates migration, adhesion and actin cytoskeleton organization in mammary tumor cells through Wnt signaling modulation. Breast Cancer Res Treat (In press).

25. Torisu Y, Watanabe A, Nonaka A, et al: Human homolog of NOTUM, overexpressed in hepatocellular carcinoma, is regulated transcriptionally by beta-catenin/TCF. Cancer Sci 99: 1139-1146, 2008

26. Jia HL, Ye QH, Qin LX, et al: Gene expression profiling reveals potential biomarkers of human hepatocellular carcinoma. Clin Cancer Res 13: 1133-1139, 2007.

27. Nakatsura T, Kageshita T, Ito S, et al: Identification of glypican-3 as a novel tumor marker for melanoma. Clin Cancer Res 10: 6612-6621, 2004.

28. Ikuta Y, Nakatsura T, Kageshita T, et al: Highly sensitive detection of melanoma at an early stage based on the increased serum secreted protein acidic and rich in cysteine and glypican-3 levels. Clin Cancer Res 11: 8079-8088, 2005.

29. Nakatsura T and Nishimura Y: Usefulness of the novel oncofetal antigen glypican-3 for diagnosis of hepatocellular carcinoma and melanoma. BioDrugs 19: 71-77, 2005.

30. Goodman ZD, Ishak KG, Langloss JM, Sesterhenn IA and Rabin L: Combined hepatocellular-cholangiocarcinoma: a histologic and immunohistochemical study. Cancer 55: 124135,1985

31. Kassahun WT and Hauss J: Management of combined hepatocellular and cholangiocarcinoma. Int J Clin Pract (In press).

32. Taketa K: Alpha-fetoprotein: reevaluation in hepatology Hepatology 12: 1420-1432, 1990.

33. Tangkijvanich $\mathrm{P}$, Tosukhowong $\mathrm{P}$, Bunyongyod $\mathrm{P}$, et al: AlphaL-fucosidase as a serum marker of hepatocellular carcinoma in Thailand. Southeast Asian J Trop Med Public Health 30: 110-114, 1999.

34. Filmus $\mathrm{J}$ and Capurro M: Glypican-3 and alphafetoprotein as diagnostic tests for hepatocellular carcinoma. Mol Diagn 8: $207-212,2004$

35. Wee A: Fine needle aspiration biopsy of the liver: algorithmic approach and current issues in the diagnosis of hepatocellular carcinoma. Cytojournal 2: 7, 2005.

36. Man XB, Tang L, Zhang BH, et al: Upregulation of Glypican-3 expression in hepatocellular carcinoma but downregulation in cholangiocarcinoma indicates its differential diagnosis value in primary liver cancers. Liver Int 25: 962-966, 2005.

37. Tickoo SK, Zee SY, Obiekwe S, et al: Combined hepatocellular-cholangiocarcinoma: a histopathologic, immunohistochemical, and in situ hybridization study. Am J Surg Pathol 26: 989-997, 2002.

38. Yano Y, Yamamoto J, Kosuge T, et al: Combined hepatocellular and cholangiocarcinoma: a clinicopathologic study of 26 resected cases. Jpn J Clin Oncol 33: 283-287, 2003

39. Nishie A, Yoshimitsu K, Asayama Y, et al: Detection of combined hepatocellular and cholangiocarcinomas on enhanced CT: comparison with histologic findings. AJR 184: 1157-1162, 2005.

40. Chu PG, Ishizawa S, Wu E and Weiss LM: Hepatocyte antigen as a marker of hepatocellular carcinoma: an immunohistochemical comparison to carcinoembryonic antigen, CD10, and alpha-fetoprotein. Am J Surg Pathol 26: 978-988, 2002.

41. Zhang F, Chen XP, Zhang W, et al: Combined hepatocellular cholangiocarcinoma originating from hepatic progenitor cells: immunohistochemical and double-fluorescence immunostaining evidence. Histopathology 52: 224-232, 2008.

42. Taguchi J, Nakashima O, Tanaka M, Hisaka T, Takazawa T and Kojiro M: A clinicopathological study on combined hepatocellular and cholangiocarcinoma. J Gastroenterol Hepatol 11: 758-764, 1996.

43. Wu PC, Fang JW, Lau VK, Lai CL, Lo CK and Lau JY: Classification of hepatocellular carcinoma according to hepatocellular and biliary differentiation markers. Clinical and biological implications. Am J Pathol 149: 1167-1175, 1996. 
44. Nakatsura T, Komori H, Kubo T, et al: Mouse homologue of a novel human oncofetal antigen, glypican-3, evokes T-cellmediated tumor rejection without autoimmune reactions in mice. Clin Cancer Res 10: 8630-8640, 2004.

45. Komori H, Nakatsura T, Senju S, et al: Identification of HLAA2- or HLA-A24-restricted CTL epitopes possibly useful for glypican-3-specific immunotherapy of hepatocellular carcinoma. Clin Cancer Res 12: 2689-2697, 2006.

46. Motomura Y, Senju S, Nakatsura T, et al: Embryonic stem cell-derived dendritic cells expressing glypican-3, a recently identified oncofetal antigen, induce protective immunity against highly metastatic mouse melanoma, B16-F10. Cancer Res 66 : 2414-2422, 2006.

47. Motomura Y, Ikuta Y, Kuronuma T, et al: HLA-A2 and -A24restricted glypican-3-derived peptide vaccine induces specific CTLs: preclinical study using mice. Int J Oncol 32: 985-990, 2008 .
48. Grozdanov PN, Yovchev MI and Dabeva MD: The oncofetal protein glypican-3 is a novel marker of hepatic progenitor/oval cells. Lab Invest 86: 1272-1284, 2006.

49. Durnez A, Verslype C, Nevens F, et al: The clinicopathological and prognostic relevance of cytokeratin 7 and 19 expression in hepatocellular carcinoma: a possible progenitor cell origin. Histopathology 49: 138-151, 2006.

50. Komuta M, Spee B, Vander Borght S, et al: Clinicopathological study on cholangiolocellular carcinoma suggesting hepatic progenitor cell origin. Hepatology 47: 1544-1556, 2008

51. Fausto N and Campbell JS: The role of hepatocytes and oval cells in liver regeneration and repopulation. Mech Dev 120: 117-130, 2003.

52. Libbrecht L: Hepatic progenitor cells in human liver tumor development. World J Gastroenterol 12: 6261-6265, 2006.

53. Alison MR, Vig P, Russo F, et al: Hepatic stem cells: from inside and outside the liver? Cell Prolif 37: 1-21, 2004. 